\title{
Long-term consumption of saponins derived from Platycodi radix (22 years old) enhances hepatic insulin sensitivity and glucose-stimulated insulin secretion in $90 \%$ pancreatectomized diabetic rats fed a high-fat diet
}

\author{
Dae Yeon Kwon ${ }^{1}$, Young Seob Kim ${ }^{2}$, Sang Mee Hong ${ }^{3}$ and Sunmin Park ${ }^{3,4 *}$ \\ ${ }^{1}$ Food Functional Research Division, Korean Food Research Institutes, Sungnam 463-746, Korea \\ ${ }^{2}$ Department of Korea Research Institute of Chemical Technology, Taejon 305-600, Korea \\ ${ }^{3}$ Department of Food and Nutrition, Hoseo University, 165 Sechul-Ri Baebang-Myun Asan-Si, Chungnam-Do 336-795, Korea \\ ${ }^{4}$ Institutes of Basic Science, Hoseo University, Asan 336-795, Korea
}

(Received 19 November 2007 - Revised 6 May 2008 - Accepted 12 May 2008 - First published online 25 June 2008)

\begin{abstract}
Crude saponins derived from Chinese Platycodi radix have been reported to prevent increases in body weight and liver TAG in mice fed a high-fat diet. We investigated the effects of an extract (PR) taken from Korean Platycodi radix, which is cultivated for 22 years in the ground (Jangsaeng doraji), and its saponins (PRS) on insulin resistance and glucose-stimulated insulin secretion in $90 \%$ pancreatectomized diabetic rats fed high-fat diets. Four groups were orally supplemented with $2 \mathrm{~g}$ PR, $0 \cdot 2 \mathrm{~g}$ PRS, $20 \mathrm{mg}$ rosiglitazone (positive control) or $0 \cdot 5 \mathrm{~g}$ cellulose (negative control) per $\mathrm{kg}$ body weight on a daily basis for 8 weeks. We found that PRS lowered body weight, visceral fat mass and serum leptin levels in pancreatectomized rats in comparison to the control. PR enhanced first- and second-phase insulin secretion while PRS stimulated only first-phase insulin secretion. Glucose infusion rates to maintain euglycaemia at hyperinsulinaemic states decreased in a descending order of rosiglitazone, PRS, PR and control, but they increased hepatic glucose output in the same order. This reduction was associated with the storage of decreased TAG and increased glycogen, which was a result of enhanced tyrosine phosphorylation of anti-insulin receptor substrate- 2 and serine ${ }^{473}$ phosphporylation of protein kinase B ( $\mathrm{PKB}, \mathrm{Akt}$ ). Improved hepatic insulin signalling led to decreased phosphoenolpyruvate carboxykinase expression and reduced hepatic glucose output accordingly. In conclusion, PRS principally improves glucose homeostasis by enhancing hepatic insulin sensitivity as a consequence of reducing fat storage and stimulating insulin signalling in diabetic rats. In addition, PR contains components that promote glucose-stimulated insulin secretion.
\end{abstract}

Platycodi radix: Jangsaeng doraji: Saponins: High-fat diets: Insulin resistance: Insulin secretion: Insulin signalling

Type 2 diabetes is a heterogeneous metabolic disorder characterized by insulin deficiency and peripheral insulin resistance ${ }^{(1,2)}$. Insulin resistance affects insulin secretion with biphasic states. When insulin resistance is initially induced in the liver, skeletal muscles and adipose tissues by certain conditions such as obesity, stress, inflammation and ageing, insulin secretion from pancreatic $\beta$-cells is sufficient to maintain normoglycaemia and thus compensate for insulin resistance $^{(3)}$. However, sustained insulin resistance causes $\beta$-cell failure and the subsequent inability to release sufficient glucose-stimulated insulin secretion to compensate for insulin resistance, which leads to type 2 diabetes $^{(2)}$.

Among peripheral tissues, the liver plays an important role in regulating glucose homeostasis through the integration of insulin, counter-regulatory hormones and neural inputs. The liver maintains the normal concentration of blood glucose within a narrow range in fasting and fed conditions because of its ability to store glucose as glycogen and produce glucose from glycogen breakdown or gluconeogenic precursors ${ }^{(4)}$. A high-fat diet and type 2 diabetes induce hepatic insulin resistance by accumulating lipids in the liver, independently of BMI or intra-abdominal obesity ${ }^{(5,6)}$. Hepatic insulin resistance is defined as the failure of insulin to suppress adequately hepatic glucose production by attenuating insulin signalling. As a result, hepatic insulin resistance induces basal and postprandial hyperglycaemia. Thus, the reduction of hepatic insulin resistance through the improvement of hepatic insulin signalling contributes to relieving diabetic symptoms.

Platycodi radix, a root of Platycodon grandiflorum, has been used traditionally as an expectorant and a remedy for bronchitis, tonsillitis, laryngitis and suppurative dermatitis in China, Japan and Korea ${ }^{(7)}$. In addition, it has been reported that Platycodi radix stimulates antioxidants, immunostimulation and anti-tumour activity and prevents hyperlipidaemia and obesity ${ }^{(8-10)}$. Platycodi radix contains triterpenoidal saponins, sterols and polysaccharides. Han et al. ${ }^{(10)}$ reported

Abbreviations: IRS, insulin receptor substrate; OGTT, oral glucose tolerance test; PEPCK, phosphoenolpyruvate carboxykinase; PR, Platycodi radix extract; PRS, crude saponins from Platycodi radix; Px, pancreatectomized; RGZ, rosiglitazone; Sham rats, sham-operated rats.

* Corresponding author: Dr Sunmin Park, Department of Food and Nutrition, fax +8241548 0670, email smpark@hoseo.edu 
that crude saponins derived from Chinese Platycodi radix prevented increases in body weight, adipose tissue weight and liver TAG in mice fed a high-fat diet. The reduction of body fat results from inhibiting intestinal absorption of dietary fat by suppressing pancreatic lipase activity. The composition of Platycodi radix is reported to differ according to the areas where it was cultivated and the length of time it was cultivated. Jangsaeng doraji, cultivated for 22 years and known as Korean Platycodi radix, has a different saponin composition from Chinese and Korean Platycodi radix (cultivated for 4 years) and it may have different actions in promoting glucose homeostasis. Therefore, our goal was to investigate the mechanism by which the extract of Jangsaeng doraji and its saponins improved glucose homeostasis. We found that they alleviated diabetic symptoms by decreasing body fat and hepatic insulin resistance and improving glucose-stimulated insulin secretion from pancreatic $\beta$-cells in a mild and non-obese type 2 diabetic animal model, $90 \%$ pancreatectomized (Px) diabetic rats, fed a high-fat diet.

\section{Materials and methods}

\section{Materials}

Jangsaeng doraji was kindly provided by Sungho Jang Saeng Doraji, Korea. The dried roots $(2 \mathrm{~kg})$ were extracted twice during $5 \mathrm{~h}$ using hot water, the extract was filtered and the filtrates were lysophilized $(460 \mathrm{~g})$. The lysophilized products (PR) and crude saponins (PRS) isolated from Platycodi radix were used as supplements to be fed to rats in the experimental study. To produce crude saponins, the lysophilized products from Jangsaeng doraji were suspended in distilled water (2 litres), and subjected to Diaion HP-20 chromatography. Crude saponins were eluted with $80 \%$ methanol after the column was washed with water and $10 \%$ methanol. After heat evaporation, crude light brown saponins ( $21 \mathrm{~g})$ were crystallized with ethanol and ethyl acetate. A study made by our colleagues using a detection method with HPLC revealed that crude saponins contained deapioplatycoside E (12.41 (SD 0.18) $\mu \mathrm{g} / \mathrm{mg}$ PRS), platycoside E (33.96 (SD 0.19) $\mu \mathrm{g} /$ mg PRS), deapioplatycodin D3 (60.9 (SD 2.11) $\mu \mathrm{g} / \mathrm{mg}$ PRS), platycodin D3 (15.57 (SD 0.65) $\mu \mathrm{g} / \mathrm{mg}$ PRS), polygalacin D2 (13.68 (SD 0.37) $\mu \mathrm{g} / \mathrm{mg}$ PRS), platycodin D2 (9.77 (SD 0.82) $\mu \mathrm{g} / \mathrm{mg}$ PRS) and platycodin D (44.25 (SD 0.16$) \mu \mathrm{g} / \mathrm{mg}$ PRS $)^{(11)}$. These components of PRS were quite different from Chinese and Korean Platycodi radix (4 years old).

\section{Animals and diets}

Male Sprague-Dawley rats, weighing 211 (SD 15) g, were housed individually in stainless steel cages in a temperatureand humidity-controlled environment $\left(23^{\circ} \mathrm{C}, 60 \%\right)$ on a normal $12 \mathrm{~h}$ light-dark cycle. All surgical and experimental procedures were performed in accordance with the recommendations found in the Guide for the Care and Use of Laboratory Animals $^{(12)}$ and approved by the Institutional Animal Care and Use Committee of Hoseo University, Korea. To generate mild type 2 diabetic experimental animals, $90 \%$ of the pancreas was removed using the Hosokawa technique ${ }^{(13)}$. After the $90 \%$ pancreatectomy, the remaining pancreas was within $2 \mathrm{~mm}$ of the common bile duct and extended from the duct to the first part of the duodenum, and the actual weight of the remainder was 10 (SD 2) \% of total pancreas at the end of the pancreatectomy procedure. Fewer than $10 \%$ of the rats died after the operation and the surviving Px rats recovered from the surgery within a week. The Px rats with serum glucose levels less than $9.4 \mathrm{mmol} / \mathrm{l}$ at a fed state were excluded from the experiment 2 weeks after surgery. The Px rats included in the experiments exhibited characteristics of mild and non-obese type 2 diabetes with insulin deficiency and insulin resistance. As they had approximately only $50 \%$ insulin secretion capacity compared to the sham-operated (Sham) rats, they developed insulin resistance ${ }^{(13,14)}$. By contrast, Sham rats had a normal glucose homeostasis and did not show any diabetic symptoms.

As was demonstrated in our previous studies, Px rats fed a high-fat diet increase their intracellular fat in peripheral tissues such as the liver, skeletal muscles and adipose tissues. A highfat diet exacerbates diabetic symptoms due to a heightening in peripheral insulin resistance and attenuation of $\beta$-cell function and mass ${ }^{(14,15)}$. In keeping with past experimental conditions, all rats freely consumed water and a high-fat diet during the 8-week experimental period. The high-fat diet consisted of (energy percentage): carbohydrates (40), protein (20) and fats (40), and was semi-purified by modifying the base with an AIN-93 formulation used in experimental diets ${ }^{(16)}$. The major carbohydrate, protein and fat sources were starch plus sugar, casein (milk protein) and shortening (CJ Co., Seoul, Korea). According to oral supplementation, the Px rats were randomly divided into four different groups of twenty animals each with comparable mean body weight. Four groups were orally supplemented with $2 \mathrm{~g}$ PR, $0 \cdot 2 \mathrm{~g}$ PRS, $20 \mathrm{mg}$ rosiglitazone (RGZ; GlaxoSmithKline, Uxbridge, UK) or $0.5 \mathrm{~g}$ cellulose (a negative control) per $\mathrm{kg}$ body weight on a daily basis. RGZ (a positive control) is a commercially available insulin sensitizer in the thiazolidinedione class.

Overnight fasted serum glucose levels, food and water intakes, and body weight were measured every Tuesday at 10.00 hours. An oral glucose tolerance test (OGTT) was performed every 3 weeks in overnight fasted animals by orally administering $2 \mathrm{~g}$ glucose $/ \mathrm{kg}$ body weight. Serum glucose and insulin were measured by tail bleeding at $0,10,20,30$, 45, 60, 90 and $120 \mathrm{~min}$ after glucose loading, and the average of the area under the curve of serum glucose and insulin was calculated. Serum glucose levels were analysed with a Glucose Analyzer II (Beckman, Palo Alto, CA, USA), and serum insulin and leptin levels were measured with an RIA kit (Linco Research, Billerica, MA, USA).

\section{Hyperglycaemic clamp}

After 7 weeks of the treatment, catheters were surgically implanted into the right carotid artery and left jugular vein of ten conscious and overnight fasted rats from each group after the anaesthetization with ketamine and xylazine. After 5-6d of implantation, a hyperglycaemic clamp was performed in free-moving and overnight fasted rats to determine insulin secretion capacity as described in previous studies $^{(14,17)}$. During the clamp, glucose was infused to maintain serum glucose levels of $5.5 \mathrm{~mm}$ above baseline and serum insulin levels were measured at designated times. After the clamp, rats were freely provided with food and water for $2 \mathrm{~d}$ 
and on the next day they were deprived of food for $16 \mathrm{~h}$. The rats were anaesthetized with the mixture of ketamine and xylazine, and human regular insulin (5 U/kg body weight) was injected through the inferior vena cava of the rats. After $10 \mathrm{~min}$ the rats were killed by decapitation and tissues were rapidly collected, frozen in liquid nitrogen and stored at $-70^{\circ} \mathrm{C}$ for further experiments. In order to determine the glycogen content in the liver, its lysates were centrifuged at $3000 \mathrm{rpm}$ for $10 \mathrm{~min}$ and the supernatants were deproteinized with $1.5 \mathrm{M}$-perchloric acid. The glycogen content was calculated from glucose concentrations derived from glycogen hydrolysed by $\alpha$-amyloglucosidase in an acid buffer ${ }^{(18)}$. TAG was extracted with chloroform-methanol $(2: 1, \mathrm{v} / \mathrm{v})$ from the liver and resuspended in pure chloroform ${ }^{(19)}$. TAG concentration was determined using a Trinder kit (Young Dong Pharm., Seoul, Korea).

\section{Euglycaemic hyperinsulinaemic clamp}

After the catheterization of the right carotid artery and left jugular vein at the seventh week of the experimental periods, a euglycaemic hyperinsulinaemic clamp was performed on fasted conscious rats to determine insulin resistance as previously described ${ }^{(3)}$. $\left[3-{ }^{3} \mathrm{H}\right]$ Glucose (NEN Life Science, Boston, MA, USA) was continuously infused during a $4 \mathrm{~h}$ period at the rate of $0.05 \mu \mathrm{Ci} / \mathrm{min}$. Basal hepatic glucose output was measured in blood collected at 100 and $120 \mathrm{~min}$ after initiation of the $\left[3-{ }^{3} \mathrm{H}\right]$ glucose infusion. Then a primed continuous infusion of human regular insulin (Humulin; Eli Lilly, Indianapolis, IN, USA) was initiated at a rate of $20 \mathrm{pmol} / \mathrm{kg}$ per min to raise plasma insulin concentration to approximately $1100 \mathrm{pM}$ at $210-240 \mathrm{~min}$. Blood samples from arteries were collected at 10 min intervals for glucose estimation, and $25 \%$ glucose was infused at variable rates as needed to clamp glucose levels at approximately $6 \mathrm{~mm}$. For the determination of plasma $\left[3-{ }^{3} \mathrm{H}\right]$ glucose concentrations, plasma was deproteinized with $\mathrm{ZnSO}_{4}$ and $\mathrm{Ba}(\mathrm{OH})_{2}$, dried to remove ${ }^{3} \mathrm{H}_{2} \mathrm{O}$, re-suspended in water, and the dpm of ${ }^{3} \mathrm{H}$ were recorded. The plasma concentration of ${ }^{3} \mathrm{H}_{2} \mathrm{O}$ was determined by the difference between ${ }^{3} \mathrm{H}$ counts without and with drying. Rates of whole-body glucose uptake and basal glucose turnover were determined as the ratio of the $\left[{ }^{3} \mathrm{H}\right]$ glucose infusion rate to the specific activity of plasma glucose $(\mathrm{dpm} / \mu \mathrm{mol})$ during the final $30 \mathrm{~min}$ of the respective experiments. Hepatic glucose production at hyperinsulinaemic clamped state was determined by subtracting the glucose infusion rate from the whole-body glucose uptake.

\section{Immunoblot analysis}

The liver collected from rats stimulated with insulin for $10 \mathrm{~min}$ was lysed with a $20 \mathrm{~mm}$-2-amino-2-hydroxymethyl1,3-propanediol buffer ( $\mathrm{pH} 7 \cdot 4$ ) containing 2 mM-EGTA, $137 \mathrm{~mm}-\mathrm{NaCl}, 1 \% \mathrm{NP} 40,10 \%$ glycerol and $12 \mathrm{~mm}-\alpha-$ glycerol phosphate and protease inhibitors. After $30 \mathrm{~min}$ on ice, the lysates were centrifuged for $10 \mathrm{~min}$ at $12000 \mathrm{rpm}$ at $4^{\circ} \mathrm{C}$. After measuring protein contents in lysate using a BioRad protein assay kit (Hercules, CA, USA), lysates with equivalent amounts of protein $(500 \mu \mathrm{g})$ were immunoprecipitated with anti-insulin receptor substrate (IRS)-2 (Upstate Biotechnology Inc., Lake Placid, NY, USA) antibodies, followed by resolving with SDS-PAGE and immunoblotted with antiphosphotyrosine protein (py20; Upstate Biotechnology Inc.). IRS2 contents were detected after stripping py20 antibody. Protein Kinase B (PKB, Akt), phospo-Akt ${ }^{\text {ser478 }}$ (Cell Signaling Technology, Beverly, MA, USA) and phosphoenolpyruvate carboxykinase (PEPCK), generously provided by Dr Garner of Vanderbilt University, were measured by immunoblotting with respective antibodies after equal amounts of protein $(30-50 \mu \mathrm{g})$ were resolved by SDS-PAGE ${ }^{(14,15)}$. The intensity of protein expression was determined using Imagequant TL (Amersham Biosciences, Piscataway, NJ, USA). These experiments were repeated three times for each group.

\section{Statistical analysis}

Statistical analysis was performed using the SAS statistical analysis program and all results are expressed as means and standard deviations. Anti-diabetic effects of PR and PRS were determined by one-way ANOVA. Significant differences in the main effects among groups were identified by Tukey tests at $P<0 \cdot 05$.

\section{Results}

Platycodi radix extract and crude saponins from Platycodi radix lower body weight and fasted glucose concentrations

Although Sham rats consumed less energy daily, their body weight was higher than that of Px rats (Table 1), due in part to urinary glucose excretion and insulin deficiency. Consistent with body weight gain, epididymal fat pads and serum leptin levels were significantly higher in Sham rats than Px rats (Table 1). With the reduction of energy intake, PR lowered body weight, visceral fat mass and serum leptin levels in Px rats, compared to the control group (Table 1). PRS, crude saponins derived from Platycodi radix, reduced body weight, visceral fat mass and serum leptin levels more than PR, which indicates that PRS was the operative component of PR with regard to changes in body weight. Moreover, the amount of saponins in PR was much less than the amount in PRS. PR may also contain a component that counteracts against PRS and so allows for weight gain.

The overnight fasted serum glucose levels of Px rats were higher than Sham rats. This increase was accompanied by a concomitant decrease in serum insulin levels (Table 1). Serum glucose and insulin levels indicated that Px rats exhibited diabetic symptoms due to the deficient insulin secretion. PR, PRS and RGZ lowered fasted glucose concentrations in $\mathrm{Px}$ rats in comparison to the control group. However, PR raised overnight fasted serum insulin levels but not PRS (Table 1). This suggested that PR might contain an unknown component to enhance insulin secretion.

\section{Platycodi radix extract and crude saponins from Platycodi radix improve glucose homeostasis}

Serum glucose levels were higher in diabetic rats by approximately $76 \%$ more than normal Sham rats during OGTT. PRS and RGZ lowered serum glucose levels at their peak in diabetic rats and quickly reduced the levels after the peak during OGTT, compared to the control (Fig. 1 (A)). PR also 
Table 1. Metabolic parameters at the end of experimental periodsł

(Mean values and standard deviations)

\begin{tabular}{|c|c|c|c|c|c|c|c|c|c|c|}
\hline & \multicolumn{8}{|c|}{ Pancreatectomized rats } & \multirow{2}{*}{\multicolumn{2}{|c|}{$\begin{array}{l}\text { Sham-operated } \\
\text { rats }(n 20)\end{array}$}} \\
\hline & \multicolumn{2}{|c|}{ Control (n 20) } & \multicolumn{2}{|c|}{$\mathrm{PR}(n 20)$} & \multicolumn{2}{|c|}{ PRS (n 20) } & \multicolumn{2}{|c|}{$\mathrm{RGZ}(n 20)$} & & \\
\hline & Mean & SD & Mean & SD & Mean & SD & Mean & SD & Mean & SD \\
\hline Body weight (g) & $393^{a}$ & 14 & $352^{b}$ & 20 & $318^{c}$ & 12 & $398^{a *}$ & 18 & 439†† & 28 \\
\hline Epididymal fat pads (g) & $5 \cdot 4^{\mathrm{a}}$ & 0.7 & $3.9^{b}$ & 0.6 & $2 \cdot 4^{\mathrm{C}}$ & 0.5 & $5 \cdot 3^{a *}$ & 0.8 & $6.4 \dagger \dagger$ & 0.9 \\
\hline $\mathrm{kJ} / \mathrm{d}$ & $496 \cdot 6^{a}$ & $54 \cdot 0$ & $396 \cdot 6^{\mathrm{b}}$ & $47 \cdot 7$ & $341 \cdot 8^{c}$ & $45 \cdot 6$ & $523 \cdot 8^{a *}$ & $55 \cdot 2$ & $344 \cdot 8 \dagger \dagger$ & $52 \cdot 3$ \\
\hline $\mathrm{kcal} / \mathrm{d}$ & $118 \cdot 7^{a}$ & $12 \cdot 9$ & $94 \cdot 8^{\mathrm{b}}$ & 11.4 & $81 \cdot 7^{\mathrm{C}}$ & $10 \cdot 9$ & $125 \cdot 2^{a \star}$ & $13 \cdot 2$ & $82.4 † \dagger$ & $12 \cdot 5$ \\
\hline Overnight fasted serum glucose (mm) & $7 \cdot 1^{\mathrm{a}}$ & 0.8 & $6 \cdot 1^{b}$ & 0.9 & $6 \cdot 0^{\mathrm{b}}$ & 0.8 & $6 \cdot 2^{b *}$ & 0.8 & 4.6†t† & 0.6 \\
\hline Overnight fasted serum insulin $(\mathrm{ng} / \mathrm{ml})$ & $0.76^{\mathrm{b}}$ & 0.13 & $0.90^{\mathrm{a}}$ & 0.12 & $0.78^{b}$ & 0.16 & $0.71^{b *}$ & $0 \cdot 16$ & $1.25+\dagger$ & 0.21 \\
\hline Overnight fasted leptin levels (ng/ml) & $5 \cdot 3^{a}$ & 0.7 & $4 \cdot 6^{\mathrm{b}}$ & 0.7 & $3 \cdot 0^{\mathrm{C}}$ & 0.6 & $5 \cdot 4^{a \star}$ & 0.7 & $6.4 \dagger \dagger$ & 0.8 \\
\hline
\end{tabular}

PR, Platycodi radix extract; PRS, crude saponins from Platycodi radix; RGZ, rosiglitazone.

a,b,c Mean values within a row with unlike superscript letters were significantly different (Tukey test; $P<0.05$ ).

* Mean values were significantly different among the groups of pancreatectomized rats (one-way ANOVA; $P<0.05$ ).

Mean values were significantly different from those of the pancreatectomized control rats (two-sample $t$ test): $\dagger \dagger P<0.01, \dagger \dagger \dagger P<0.001$.

$\ddagger$ For details of procedures, see Materials and methods.

increased serum glucose levels in a similar manner to PRS up until $40 \mathrm{~min}$, but after $50 \mathrm{~min}$, PR decreased serum glucose levels at a slower rate than PRS. Areas under the curves of serum glucose and insulin during OGTT are shown in Fig. 1 (B). Px rats exhibited a much bigger area under the curve of glucose during OGTT, in comparison with Sham rats $(P<0 \cdot 01)$. This increase was explained by the reduction of the area under the curve of insulin in Px rats, compared to Sham rats (Fig. 1 (B)). After the oral glucose load, the administration of PRS in Px rats resulted in a significantly lower area under the curve of serum glucose $(24 \%)$, compared to the control group $(P<0.05)$. This decrease was comparable to Px rats administered with RGZ as a positive control, but the area did not reach the levels of Sham rats. PR also decreased the area under the curve of glucose in comparison with the control group, but its area was higher than PRS (Fig. 1 (B)). PR displayed a higher total incremental area under the insulin curves $(P<0 \cdot 05)$. PRS did not elevate the area of serum insulin significantly compared to the control, while RGZ rather decreased it (Fig. 1 (B)). The results of OGTT demonstrated that PR and PRS might regulate glucose homeostasis for the most part in a different manner. PR might contain a component to improve insulin secretion capacity in order to enhance glucose regulation in Px rats while PRS might act to improve insulin sensitivity. Thus, PR contained a component to counteract with PRS, which was the major component of PR.

Platycodi radix extract and crude saponins from Platycodi radix improve first-phase insulin secretion

To confirm $\beta$-cell function, a hyperglycaemic clamp was performed on rats administered PR, PRS and RGZ. During the hyperglycaemic clamp, serum insulin levels peaked between 2 and $5 \mathrm{~min}$ and then declined to a nadir at $10 \mathrm{~min}$, when glucose levels remained elevated and stable. This is known as first-phase insulin secretion. An ascending second phase of plasma insulin was observed at $60-120 \mathrm{~min}$ in all rats. Serum insulin levels in the first and second phases represent insulin secretion capacity. PR and PRS elevated first-phase insulin secretion in comparison to the control, but RGZ decreased it (Table 2). However, second-phase insulin secretion was strengthened only by PR. Due to increased second-phase insulin secretion during 60-120 min, the area under the curve of serum insulin was greater in the PR group than the other groups (Table 2). Thus, this suggests that PR promotes a glucose-stimulated insulin secretion capacity in diabetic rats and that PRS had less effect on insulin secretion capacity compared to PR.

Glucose infusion rates in the hyperglycaemic clamp indicated $\beta$-cell function and insulin sensitivity at hyperglycaemic state, calculated as the ratio of glucose infusion rates to steadystate serum insulin levels ${ }^{(20)}$. Glucose infusion rates to maintain serum glucose levels at $5.5 \mathrm{~mm}$ above baseline were found to be greater in the ascending order of the control, PR, PRS and RGZ groups (Table 2). Insulin sensitivity at hyperglycaemic state showed a comparable pattern of glucose infusion rates at the hyperglycaemic clamp. Therefore, the results of the hyperglycaemic clamp revealed that PR and PRS improved glucose homeostasis by independent pathways at hyperglycaemic states: PRS and RGZ enhanced it by improving insulin sensitivity, while PR enhanced it by promoting glucose-stimulated insulin secretion capacity (Table 2 ).

\section{Platycodi radix extract and crude saponins from Platycodi radix improve insulin sensitivity}

During a hyperinsulinaemic euglycaemic clamp, whole-body glucose utilization was measured to see whether the improved glucose tolerance of PR- and PRS-administered rats was associated with ameliorate insulin resistance. As depicted in Fig. 2 (A), glucose infusion rates and glucose uptake at about $1100 \mathrm{pM}$-serum insulin levels were lower in Px rats than Sham rats. In comparison with cellulose-administered rats, PR- and PRS-administered rats displayed increased glucose infusion rates at hyperinsulinaemic clamped states by 16.4 and $39.8 \%$, respectively. However, the increases brought on with the supplementation of PR and PRS did not reach the levels of RGZ-administered Px rats (Fig. 2 (A)). Hepatic glucose output in basal and hyperinsulinaemic clamped states 

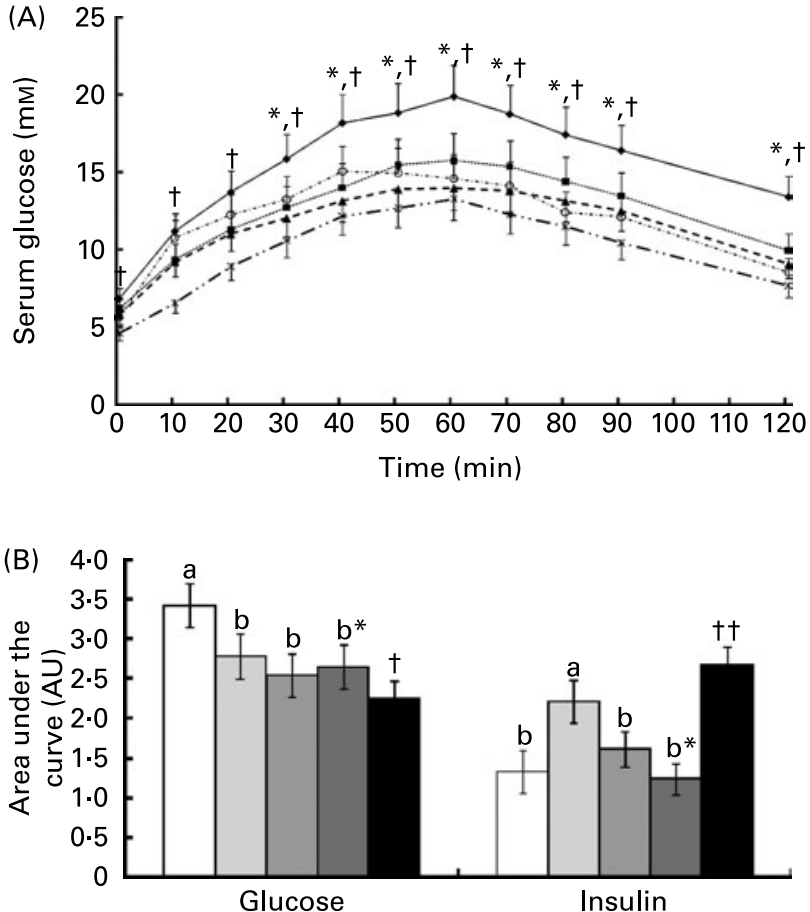

Fig. 1. The changes in serum glucose and insulin levels during oral glucose tolerance test (OGTT). During a 7-week treatment period of administering Jangsaeng doraji extract (PR), crude saponins from Jangsaeng doraji (PRS), rosiglitazone (RGZ) and water (Cont, control), an OGTT was performed in overnight fasted rats by orally administering $2 \mathrm{~g}$ glucose $/ \mathrm{kg}$. (A), Serum glucose was measured at $0,10,20,30,45,60,90$ and $120 \mathrm{~min}$ after glucose loading by tail bleeding $(-\bullet$, PR; ......, PRS; -4-, RGZ; -.o., Cont; -*-, sham-operated). (B), The average of the area under the curve of glucose and insulin was calculated in normal and diabetic rats, respectively ( $\square$, PR; $\square$, PRS; $\square, R G Z$;

$\square$, Cont; $\mathbf{\square}$, sham-operated). The sample size in each group was the same as in Table 2. Values are means with their standard deviations depicted by vertical bars. Mean values were significantly different among the groups of pancreatomized rats: ${ }^{*} P<0.05$. Mean values were significantly different from those of the pancreatectomized control rats: $\dagger P<0.05, \dagger \dagger P<0.01$. ${ }^{\mathrm{a}, \mathrm{b}, \mathrm{c}}$ Mean values with unlike superscript letters were significantly different $(P<0.05)$.

was not suppressed in Px rats as much as in Sham rats (Fig. 2 (B)). Consistent with overnight fasted glucose levels, PR, PRS and RGZ tended to lower basal hepatic glucose output, but they were not significantly different. Since glucose uptake was similar among the different groups, a good portion of the difference in glucose infusion rates could be accounted for by the insulin-stimulated reduction in hepatic glucose production, which represents attenuated hepatic insulin sensitivity. Hepatic glucose output in hyperinsulinaemic states was suppressed in the ascending order of RGZ, PRS, PR and cellulose (Fig. 2 (B)). PRS improved hepatic insulin action better than PR. PRS increased glycogen storage in the liver of the Px rats and decreased their TAG contents at the end of the experimental period (Fig. 2 (C)). PR exhibited the same trends of PRS in hepatic glycogen and TAG but the changes were minimal. These changes explained the decrease of hepatic glucose output in a hyperinsulinaemic clamp state.

Platycodi radix extract and crude saponins from Platycodi radix enhance hepatic insulin signalling

Since the changes in hepatic glucose homeostasis can be related to the modification of hepatic insulin signalling, the

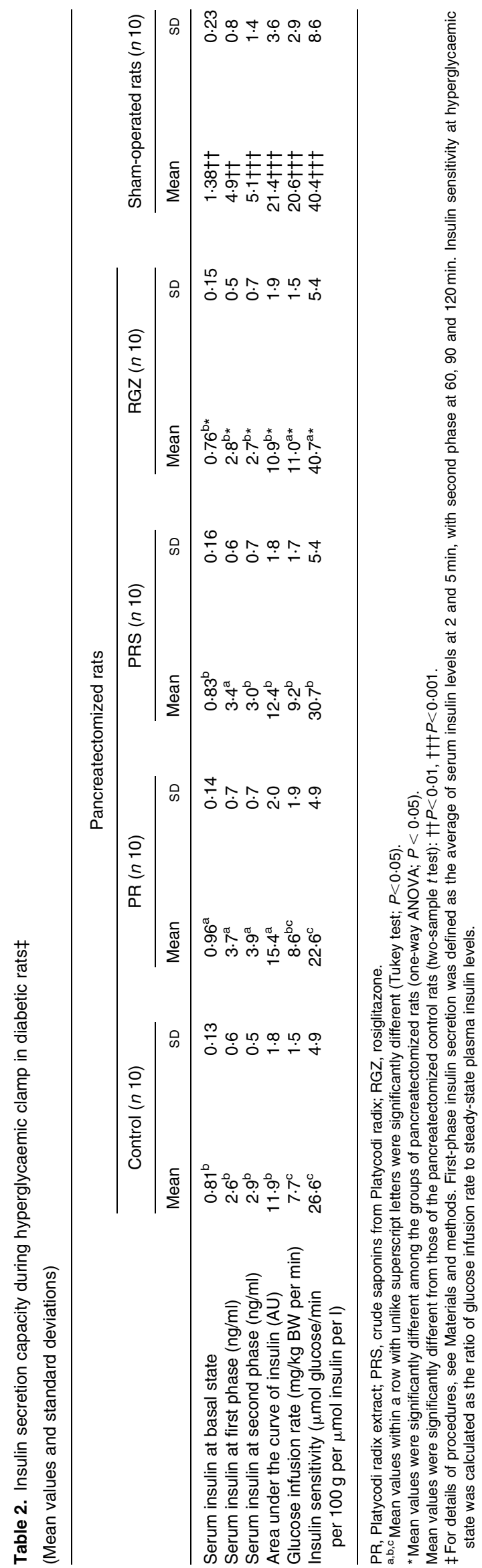


(A)

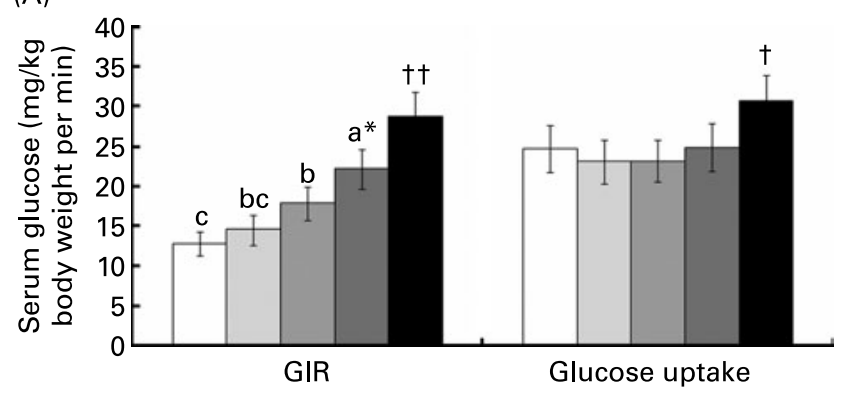

(B)

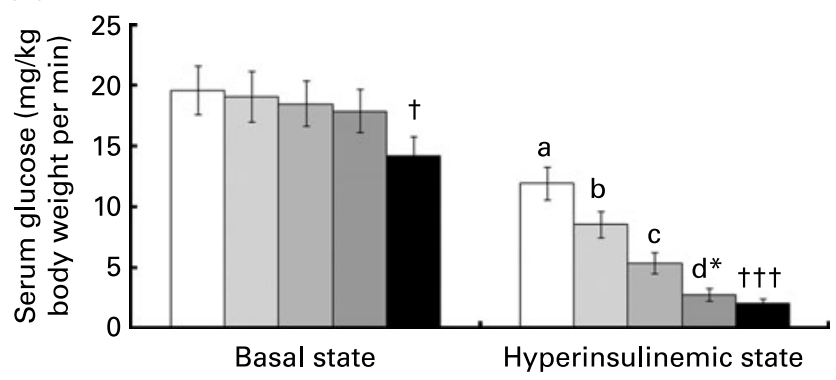

(C)

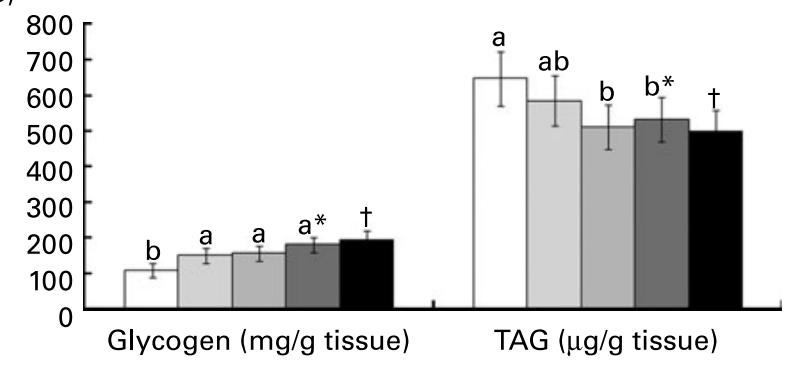

Fig. 2. Serum glucose and insulin levels, and glucose infusion rates (GIR) during a euglycaemic hyperinsulinemic clamp. After an 8-week treatment period of Platycodi radix extract (ㄷ), crude saponins from Platycodi radix (ㄷ), rosiglitazone ( $\square$ ) and water ( $\square$, control), a euglycaemic hyperinsulinemic clamp was performed in conscious, free-moving and overnight fasted rats to determine whole-body insulin resistance ( $\mathbf{\square}$, sham-operated). (A), GIR and glucose uptake at a clamped steady-state. (B), Hepatic glucose output at baseline and hyperinsulinaemic state about $1100 \mathrm{pm}$-serum insulin. (C) Hepatic glycogen and TAG contents. Mean values were significantly different among the groups of pancreatomized rats: ${ }^{\star} P<0.05$. Mean values were significantly different from those of the pancreatectomized control rats: $\dagger P<0.05, \dagger^{\prime} P<0.01, \dagger_{\dagger}+P<0.001$. ${ }^{a, b, c, d}$ Mean values with unlike superscript letters were significantly different $(P<0 \cdot 05)$.

signalling was examined in order to investigate the molecular pathways. Tyrosine phosphorylation of IRS2 and serine phosphorylation of Akt were potentiated in Px rats fed with PRS in comparison to the control rats (Fig. 3). PR also increased their phosphorylation but not as much as PRS. The potentiation of their phosphorylation in PRS was similar to that in RGZ. Among the treatments there was no visible difference in the protein levels of IRS2 and Akt. In parallel with the intensity of Akt phosphorylation, PEPCK expression was reduced in Px rats administered with PRS and RGZ in comparison to the control (Fig. 3). In parallel with the intensity of IRS2 and Akt phosphorylation, PEPCK expression of PR was higher than that of PRS but it was lower than that of the control. Decreased expression of PEPCK indirectly implied
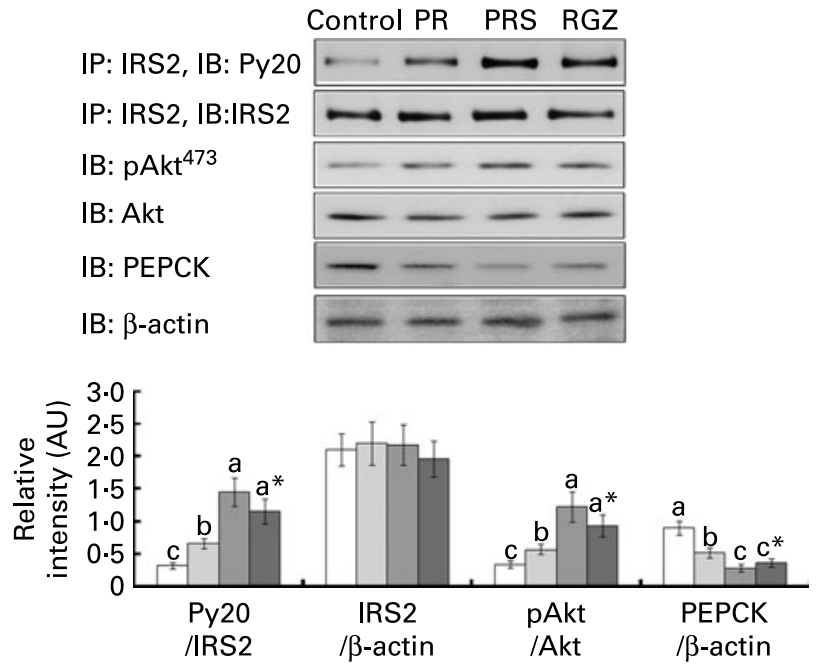

Fig. 3. The modulation of insulin signalling in the liver at the end of experimental periods. After $10 \mathrm{~min}$ of insulin $(5 \mathrm{U} / \mathrm{kg}$ body weight) stimulation through the inferior vena cava at the end of each experimental period, the liver collected from the rats administered with Platycodi radix extract $(\square, P R)$, crude saponins from Platycodi radix ( $\square$, PRS), rosiglitazone ( $\square, R G Z$ ) and water ( $\square$, control) for 8 weeks was immediately lysed with a lysis buffer. The phosphorylation and expression levels of the insulin receptor substrate 2 (IRS2), protein kinase B (PKB, Akt) and phosphoenolpyruvate carboxykinase (PEPCK), involved in insulin signalling, were determined by immunoprecipitation (IP) and immunoblotting (IB) with specific antibodies. The intensity of protein expression was determined using an Imagequant TL (Amersham Biosciences, Piscataway, NJ, USA). These experiments were repeated four times for the liver. Values are means with their standard deviations depicted by vertical bars. Mean values were significantly different among the groups of pancreatomized rats: ${ }^{\star} P<0.05$. ${ }^{\mathrm{a}, \mathrm{b}, \mathrm{c}}$ Mean values with unlike superscript letters were significantly different $(P<0.05)$.

an enhanced insulin signalling cascade in the liver and resulted in the suppression of hepatic glucose output during the hyperinsulinaemic clamp state. In addition, the stimulation of hepatic insulin signalling promoted glucose utilization in the liver, which was accounted for by an increased glycogen accumulation and decreased lipid storage.

\section{Discussion}

It has been reported that Platycodi radix has an anti-obesity property that prevents mice fed a high-fat diet from becoming obese $^{(10)}$. Saponins are regarded as the major component in Platycodi radix, and they are attributed with alleviating metabolic syndrome ${ }^{(8-10)}$. However, Platycodi radix also contains triterpene (betulin), polysaccharides (inulin) and phytosterols, which may have a beneficial effect on metabolic diseases. In the present study, we focused on studying the anti-diabetic effects of PR and PRS in diabetic Px rats fed a high-fat diet since their modus operandi have not been studied in nonobese diabetic rats. PR contained crude saponins and other active components that may co-operate and/or counteract with the crude saponins. We found that PR and PRS decreased visceral fat and body weight and improved glucose homeostasis in diabetic rats fed a high-fat diet. PRS mainly enhanced glucose homeostasis by activating whole-body glucose disposal rates, denoting an improvement in peripheral insulin sensitivity, including the liver, while PR additionally contained the components to potentiate glucose-stimulated insulin secretion. 
Han et al. ${ }^{(21)}$ revealed that rats fed a high-fat diet plus crude saponins (isolated from Chinese Platycodi radix) showed a reduction in the small intestinal absorption of dietary fat by inhibiting pancreatic lipase activity and also decreased their body weight and adipose tissues more than those rats fed only a high-fat diet. Furthermore, crude saponins significantly decreased the hepatic TAG and total cholesterol concentrations without decreasing energy intake. This was explained by the platycodin D contained in Chinese Platycodi radix, which inhibits pancreatic lipase in vitro to reduce fat absorption $^{(21)}$. Since the suppression of fat absorption is part of the lipid metabolism, the involvement of fat oxidation and/or fat synthesis in the reduction of hepatic TAG storage cannot be ignored. However, fat was not excreted into the faeces of rats fed a high-fat diet containing PR and PRS in the present study (data not shown) even though body fat, including hepatic lipid storage, was reduced. This suggests that the saponins modulate fat utilization in the tissues. These differences between Jangsaeng doraji and Chinese Platycodi radix may originate from the different composition of the crude saponins. Our previous study revealed that Jangsaeng doraji contained more deapioplatycoside E, platycoside E, deapioplatycodin D3, platycodin D3, polygalacin D2, platycodin D2 than Chinese Platycodi radix by $2-9$-fold but had less than half the amount of platycodin D compared to its Chinese counterpart ${ }^{(11)}$. In addition, most of the saponins contained in Jangsaeng doraji were present in higher quantities than those in the original Korean Platycodi radix (4 years old) by 40-300\%. In particular, Jangsaeng doraji contained much more polygalacin D2 than the Chinese variety and the original Korean Platycodi radix (4 years old). In the future we need to study which saponins have the anti-diabetic and anti-obesity properties.

Insulin resistance refers to a decreased capacity of circulating insulin to regulate nutrient metabolism, especially glucose utilization $^{(22,23)}$. Animals, including man, produce hyperinsulinaemia to normalize blood glucose levels in an insulinresistant state but they do not develop diabetes, at least in part, due to compensation by pancreatic $\beta$-cells ${ }^{(23,24)}$. Animals become diabetic when insulin secretion does not compensate for insulin resistance. Px rats are an appropriate animal model for non-obese and insulin-deficienct type 2 diabetic rats to study whether herbal extracts have anti-diabetic actions to improve peripheral insulin resistance and insulin secretion. Px rats did not increase their body weight, body fat and serum leptin levels with a high-fat diet as much as normal rats. This is connected to the low levels of serum insulin, which decreases fat and leptin synthesis in adipose tissues ${ }^{(25,26)}$. Furthermore, reduced body fat lowers serum leptin levels ${ }^{(25)}$. Even though Px rats have less body fat and a smaller body weight than Sham rats, both body fat and weight are increased with a high-fat diet. A high-fat diet attenuates leptin action in the hypothalamus for the long-term regulation of food intake, which dysregulates food intake and leads to increased body $\mathrm{fat}^{(25)}$. Px rats fed a high-fat diet increase body fat and serum leptin levels more than those fed a low-fat diet. Thus, Px rats fed a high-fat diet can be used for investigating body fat regulation as a mild non-obese diabetic animal model. In the present study, body fat and serum leptin levels were lower in Px rats than Sham rats, consistent with other findings ${ }^{(25,26)}$. In parallel with energy intake, PR and PRS reduced body fat and serum leptin levels, compared to the control in Px rats fed a high-fat diet. The administration of RGZ in Px rats did not increase body fat and serum leptin levels more than the control in the present study. However, the effects of RGZ on body fat and serum leptin levels remain controversial in various situations ${ }^{(27,28)}$.

The liver is central to nutrient homeostasis and stores glucose after a meal as glycogen or converts excess glucose to fatty acids and produces glucose during fasting. The integration of insulin signals with those generated by counter-regulatory hormones and neuronal inputs establishes a dynamic network that co-ordinates systemic nutrient homeostasis ${ }^{(1,29)}$. Dysregulation of hepatic glucose and lipid metabolism was associated with metabolic syndrome and diabetes. In the liver, IRS2 is predominant among IRS proteins and it is an important regulatory point for insulin signalling cascading in hepatocytes ${ }^{(30,31)}$. Cell-based experimentation with immortalized hepatocytes suggests that IRS2 is the major cause of both the metabolic and the growth-promoting actions of insulin $^{(30)}$. Moreover, IRS2 signalling is essential for the suppression of gluconeogenesis and apoptosis in immortalized neonatal hepatocytes ${ }^{(32,33)}$. Activated IRS2 contributes to the serine ${ }^{473}$ phosphorylation of Akt, which is essential in controlling hepatic gluconeogenesis, as suggested by the complete loss of Akt phosphorylation in IRS2 liver-specific knockout mice with a background of IRS1 whole-body knockout mice ${ }^{(32)}$. In these mice, changes in hepatic gene expression strongly favour hepatic glucose production, including increased glucose 6-phosphatase and PEPCK, and decreased glucokinase. Our preliminary study revealed that hepatic insulin signalling was substantially more attenuated in Px rats than Sham rats (data not shown). In this present study, PRS potentiated IRS2(Akt signalling in the liver, which resulted in PEPCK expression to suppress gluconeogenesis in a hyperinsulinaemic state. This demonstrated that PRS improved hepatic insulin action though potentiating insulin signalling.

Although hepatic insulin sensitivity was markedly enhanced and hepatic glucose output was decreased in RGZ-treated rats more than PRS-treated rats, in the present study RGZ enhanced hepatic insulin signalling at hyperinsulinaemic states just as much as PRS. This suggests that the improvement in hepatic insulin action caused by RGZ may involve other pathways besides hepatic insulin signalling. In addition, PR promoted insulin signalling, but not as much as PRS, which suggests that the amount of saponins in PR was less than the amount in PRS. This indicates that PR may contain a component that counteracts against PRS and causes hepatic insulin signalling to be inhibited. PR might contain unknown components, such as triterpene, polysaccharides and phytosterols, that offset the PRS effect of promoting hepatic insulin signalling. By contrast, it was shown in the present study that PR components, except PRS, ameliorated glucose homeostasis by enhancing glucose-stimulated insulin secretion. Therefore, PR contained PRS and other components that differently regulated glucose metabolism in Px diabetic rats.

In addition, fatty liver and hepatic TAG accumulation are strongly associated with obesity and type 2 diabetes, and are subjected to nutritional influences. The accumulation of TAG in the liver increases insulin resistance and oxidative stress through decreased fatty acid oxidation and increased lipogenesis ${ }^{(34)}$. Hepatic regulation of glucose and lipid 
homeostasis is influenced by a complex system of hormones and hormonally related signalling pathways ${ }^{(29)}$. Insulin signalling is one of several hormonal signalling pathways in the liver, of which the improvement is associated with decreased hepatic TAG accumulation. Liver-specific insulin receptor knockout mice are glucose intolerant but not diabetic and they develop only mild hepatic steatosis ${ }^{(35)}$. However, even a partial knockdown of IRS1 and IRS2 by short hairpin RNA adenovirus caused glucose intolerance and hepatic steatosis ${ }^{(36)}$. Thus, IRS1 and IRS2 play an important role in maintaining hepatic glucose and lipid metabolism. The present study showed that PRS reduced hepatic TAG storage and enhanced IRS2(Akt signalling, which affected the reduction of hepatic glucose output. However, PR did not alter the storage compared to the control, but it did reduce hepatic glucose output and improved insulin signalling. Thus, the contents of hepatic TAG partly affect the promotion of hepatic insulin sensitivity.

In summary, PRS, crude saponins extracted from Jangsaeng doraji, lowered body weight, visceral fat mass and hepatic TAG storage in Px rats fed a high-fat diet in comparison to the control in the present study. The reduction of body fat by the saponins was related to the enhancement of fat utilization. PRS increased whole-body glucose disposal rates in diabetic rats and reduced hepatic glucose output at hyperinsulinaemic clamped states, compared to the control group. The improvement of peripheral insulin resistance by PRS potentiated only first-phase insulin secretion in diabetic rats. Unlike PRS, PR enhanced both first and second phases of insulin secretion. The reduction of hepatic glucose production was associated with the decrease of PEPCK expression, which resulted from the enhancement of hepatic insulin signalling (tyrosine phosphorylation of IRS2 $\rightarrow$ serine $^{473}$ phosphporylation of Akt). In conclusion, PRS was the functional component of PR that enhanced hepatic glucose metabolism in diabetic rats, while PR contained unknown components to promote glucose-stimulated insulin secretion.

\section{Acknowledgements}

This study was supported in part by the Inter-Institutional Collaboration Research Program under the Korea Research Council for Industrial Science \& Technology (KOCI), Korea. PEPCK antibody was generously provided by Dr Garnner of Vanderbilt University. There are no conflicts of interest. D. Y. K. contributed to the design of experimental study and data analysis; Y. S. K. prepared the extract and saponins from Jangsaeng doraji; S. M. H. performed data analysis; S. P. prepared the manuscript.

\section{References}

1. DeFronzo RA \& Ferrannini E (2001) Regulation of intermediary metabolism during fasting and feeding. In Endocrinology, pp. 737-775 [LJ DeGroot and JL Jameson, editors]. Philadelphia, PA: W.B. Saunders Co.

2. Taylor SI (1999) Deconstructing type 2 diabetes. Cell 97, 9-12.

3. Kim JK, Kim YJ, Fillmore JJ, et al. (2001) Prevention of fat-induced insulin resistance by salicylate. J Clin Invest 108, 437-446.

4. Rutter GA (2000) Diabetes: the importance of the liver. Curr Biol 10, R736-R738.
5. Dong X, Park S, Lin X, Copps K, Yi X \& White MF (2006) Irs1 and Irs2 signaling is essential for hepatic glucose homeostasis and systemic growth. $J$ Clin Invest 116, 101-114.

6. Flowers MT, Miyazaki M, Liu X \& Ntambi JM (2006) Probing the role of stearoyl-CoA desaturase-1 in hepatic insulin resistance. J Clin Invest 116, 1478-1481.

7. Shin CY, Lee WJ, Lee EB, Choi EY \& Ko KH (2002) Platycodin D and D3 increase airway mucin release in vivo and in vitro in rats and hamsters. Planta Med 68, 221-225.

8. Choi CY, Kim JY, Kim YS, Chung YC, Seo JK \& Jeong HG (2001) Aqueous extract isolated from Platycodon grandiflorum elicits the release of nitric oxide and tumor necrosis factor-a from murine macrophages. Int Immunopharmacol 1, $1141-1151$.

9. Kim KS, Seo EK, Lee YC, Lee TK, Cho YW, Ezaki O \& Kim CH (2000) Effect of dietary Platycodon grandiflorum on the improvement of insulin resistance in obese Zucker rats. J Nutr Biochem 11, 420-424.

10. Han LK, Xu BJ, Kimura Y, Zheng Y \& Okuda H (2000) Platycodi radix affects lipid metabolism in mice with high fat dietinduced obesity. J Nutr 130, 2760-2764.

11. Kim HK, Choi JS, Yoo DS, et al. (2007) HPLC analysis of saponins in Platycodi Radix. Kr J Pharmacognosy 38, 1-18.

12. National Research Council (1996) Guide for the Care and Use of Laboratory Animals. Bethesda, MD: National Institutes of Health.

13. Hosokawa YA, Hosokawa H, Chen C \& Leahy JL (1996) Mechanism of impaired glucose-potentiated insulin secretion in diabetic $90 \%$ pancreatectomy rats. Study using glucagons like peptide-1 (7-37). J Clin Invest 97, 180-186.

14. Choi SB, Jang JS \& Park S (2005) Estrogen and exercise may enhance beta-cell function and mass via insulin receptor substrate 2 induction in ovariectomized diabetic rats. Endocrinology 146, 4786-4794.

15. Kwon DY, Jang JS, Hong SM, Lee JE, Sung SR, Park HR \& Park S (2007) Long-term consumption of fermented soybeanderived Chungkookjang enhances insulinotropic action unlike soybeans in $90 \%$ pancreatectomized diabetic rats. Eur J Nutr 46, 44-52.

16. American Institute of Nutrition (1993) AIN-93 purified diets for laboratory rodents: final report of the American Institute of Nutrition ad hoc writing committee on the reformulation of the AIN-76A rodent diet. J Nutr 123, 1939-1951.

17. Rossetti L, Shulman GI, Zawalich W \& DeFronzo RA (1987) Effect of chronic hyperglycemia on in vivo insulin secretion in partially pancreatectomized rats. $J$ Clin Invest $\mathbf{8 0}$, 1037-1044.

18. Frontoni S, Choi SB, Banduch D, Rossetti L, et al. (1991) In vivo insulin resistance induced by amylin primarily through inhibition of insulin-stimulated glycogen synthesis in skeletal muscle. Diabetes 40, 568-573.

19. Sebokova E, Klimes I, Moss R, Stolba P, Wiersma MM \& Mitkova A (1993) Muscle GLUT 4 protein levels and impaired triglyceride metabolism in streptozotocin diabetic rats. Effect of a high sucrose diet and fish oil supplementation. Ann N Y Acad Sci 683, 218-227.

20. Muzumdar R, Ma X, Atzmon G, Vuguin P, Yang X \& Barzilai N (2004) Decrease in glucose-stimulated insulin secretion with aging is independent of insulin action. Diabetes 53, 441-446.

21. Han LK, Zheng YN, Xu BJ, Okuda H \& Kimura Y (2002) Saponins from platycodi radix ameliorate high fat diet-induced obesity in mice. J Nutr 132, 2241-2245.

22. Okamoto H, Obici S, Accili D \& Rossetti L (2005) Restoration of liver insulin signaling in Insr knockout mice fails to normalize hepatic insulin action. J Clin Invest 115, 1314-1322.

23. Abdul-Ghani MA, Tripathy D \& DeFronzo RA (2006) Contributions of beta-cell dysfunction and insulin resistance to the 
pathogenesis of impaired glucose tolerance and impaired fasting glucose. Diabetes Care 29, 1130-1139.

24. Obici S, Feng Z, Karkanias G, Baskin DG \& Rossetti L (2002) Decreasing hypothalamic insulin receptors causes hyperphagia and insulin resistance in rats. Nat Neurosci 5, 566-572.

25. Ahima RS, Qi Y, Singhal NS, Jackson MB \& Scherer PE (2006) Brain adipocytokine action and metabolic regulation. Diabetes 55, S145-S154.

26. Ahima RS \& Flier JS (2000) Leptin. Annu Rev Physiol 62, 413-437.

27. Johnson JA, Trasino SE, Ferrante AW Jr \& Vasselli JR (2007) Prolonged decrease of adipocyte size after rosiglitazone treatment in high- and low-fat-fed rats. Obesity (Silver Spring) 15, $2653-2663$.

28. Joosen AM, Bakker AH, Gering MJ \& Westerterp KR (2006) The effect of the PPARgamma ligand rosiglitazone on energy balance regulation. Diabetes Metab Res Rev 22, 204-210.

29. DeFronzo RA (2004) Pathogenesis of type 2 diabetes mellitus. Med Clin North Am 88, 787-835.

30. Rother KI, Imai Y, Caruso M, Beguinot F, Formisano P \& Accili D (1998) Evidence that IRS-2 phosphorylation is required for insulin action in hepatocytes. J Biol Chem 273, $17491-17497$.

31. Ide T, Shimano H, Yahagi N, et al. (2004) SREBPs suppress IRS-2-mediated insulin signalling in the liver. Nat Cell Biol 6, $351-357$.

32. Valverde AM, Burks DJ, Fabregat I, Fisher TL, Carretero J, White MF \& Benito M (2003) Molecular mechanisms of insulin resistance in IRS-2-deficient hepatocytes. Diabetes 52, 2239-2248.

33. Valverde AM, Fabregat I, Burks DJ, White MF \& Benito M (2004) IRS-2 mediates the antiapoptotic effect of insulin in neonatal hepatocytes. Hepatology 40, 1285-1294.

34. Utzschneider KM \& Kahn SE (2006) Review: the role of insulin resistance in nonalcoholic fatty liver disease. J Clin Endocrinol Metab 91, 4753-4761.

35. Michael MD, Kulkarni RN, Postic C, Previs SF, Shulman GI, Magnuson MA \& Kahn CR (2000) Loss of insulin signaling in hepatocytes leads to severe insulin resistance and progressive hepatic dysfunction. Mol Cell 6, 87-97.

36. Taniguchi CM, Ueki K \& Kahn R (2005) Complementary roles of IRS-1 and IRS-2 in the hepatic regulation of metabolism. $J$ Clin Invest 115, 718-727. 